\title{
An Improved Fuzzy Borda Count and its Application to Watershed Management
}

\author{
Jin Zou', a , Zeyuan Qiü,b \\ ${ }^{1}$ Kunming University of Science and Technology, Department of Hydraulic Engineering, Kunming, \\ China. \\ ${ }^{2} \mathrm{New}$ Jersey Institute of Technology, Department of Chemistry and Environmental Science, \\ Newark, NJ, United States \\ aJonezou@hotmail.com, 'eyuan.qiu@njit.edu
}

\begin{abstract}
Keywords: Linguistic preferences, Fuzzy, Borda count, Pairwise comparison, Group decision making, Multi- criteria decision making

Abstract. Analytic Hierarchy Process (AHP) is a popular approach to obtain the weights of criteria in Multi-criteria Decision Making (MCDM) by pairwise comparison, in which, however, if the consistency check at the final stage could not be passed, another round of judgment has to be made until the requirement is met. While in reality, especially in Group Decision Making (GDM), it is difficult to require the respondents to give their opinions once again and again. Therefore, methods to derive weights directly from the judgment matrices become imperative. The paper aims to present an approach of such a kind, which not only expands the traditional use of Borda count to take the respondents' confidence and importance into account, but also provides a way to find the preference weights of criteria. Finally, an example of its application in a real investigation about conservation buffers in a watershed is given to show its effectiveness.
\end{abstract}

\section{Introduction}

Multi-criteria decision making (MCDM) is regarded as a main part of modern decision science and operational research, which contains multiple decision criteria and multiple decision alternatives. The objective of MCDM is to find the most desirable alternative(s) from a set of available alternatives versus the selected criteria. Since the results usually differ with different choices of criteria weights, how to decide criteria weights becomes one of the most important issues that have to be settled first.

There are a lot of methods to determine the weights of criteria [1-7], and $\mathrm{Xu}$ [7] summarized different methods to obtain the order weights. Generally, they can be divided into three categories. In the first one, weights are given by a decision maker subjectively. Second, they are obtained by an objective method. Third, the weights from the subjective and objective methods may be combined. When experts make decision subjectively, they may choose different forms: (1) directly point out the value of every weight in a crisp number ( such as 0.1 ), fuzzy number ( such as $(0,0.1,0.2)$ ), or linguistic word (such as "very important"); (2) make paired comparison, in crisp, fuzzy or linguistic variable, to show that one criterion is more/less important than another; (3) give some rules to decide the weights, for example, $w_{2}>w_{1} \geq 3 w_{3}$. In this situation, it is more suitable to obtain the weights by means of optimization. Within these three forms, the second one is widely accepted because it is convenient and easier for human to make decision.

Analytic Hierarchy Process (AHP), originated by Saaty [8-9], is a popular approach to obtain the weights of criteria in MCDM by pairwise comparison. While one of its shortcomings is that if the consistency check at the final stage could not be passed, another round of judgment has to be made until the requirement is met. However, in reality, especially in Group Decision Making (GDM), it is difficult to require the respondents to give their opinions again and again. Therefore, methods to educe weights directly from the judgment matrices become imperative.

The Borda rule is an appropriate procedure in multi-person decision making when several alternatives are considered. This estimation relies on the processed information from the whole set of alternatives, not only from each agent's most preferred one [10]. In addition, Black [11, 12], Mueller 
[13] have noted that the Borda count chooses the alternative which stands highest on average in the agents' preference orderings.

The literature about the Borda rule is very extensive. After its first presentation by Borda [14], more comprehensive analysis have been made, including adjustments in the case of indifference between alternatives $[11,12,15]$, extensions to linguistic or fuzzy environment $[16,17,18]$. While García-Lapresta [18] extended Borda count into linguistic context, however, he did not take the agents' confidence and importance into consideration, and didn't provide a way to derive the values of weights from Borda count. Therefore, this paper aims to advance this method and apply it to a real investigation, whose objective is to decide the weights of five factors that affect the conservation buffers in water resources management.

The paper is organized as follows. In Section 2, some preliminary knowledge about Borda count is stated. In section 3, details of this method are described. In section 4, a real example is given to show its effectiveness. Finally, section 5 contains some concluding remarks.

\section{Linguistic Labels and Borda Count}

When making comparison, people prefer to use linguistic labels, such as very important, important, less important, etc. These linguistic labels can be expressed by fuzzy numbers and then easily transformed into numerical values to make decision. Lapresta defined a set of such linguistic labels, on which Borda count was computed [18], as stated below.

Let $L=\left\{l_{0}, l_{1} \mathrm{~K}, l_{s}\right\}$ is a set of linguistic labels, where $s \geq 2$, ranked by a linear order: $l_{0}<l_{1}<\mathrm{K}<l_{s}$. There ought to be an intermediate label representing indifference, and the rest of labels are defined around it symmetrically. An example of nine linguistic labels is shown in Table 1, wherein column 1 lists the symbols of the labels, and column 2 gives the meaning of each label.

Table 1 Semantics with nine linguistic labels

\begin{tabular}{|c|l|l|r|}
\hline Lable & \multicolumn{1}{|c|}{ Meaning } & \multicolumn{1}{|c|}{ TFN } & $V(t)$ \\
\hline$l_{0}$ & Absolutely less important & $(0,0,0,0)$ & 0.0417 \\
\hline$l_{1}$ & Much less important & $(0,0.02,0.05,0.11)$ & 0.1433 \\
\hline$l_{2}$ & Somewhat less important & $(0.05,0.11,0.17,0.25)$ & 0.2983 \\
\hline$l_{3}$ & Less important & $(0.17,0.25,0.34,0.44)$ & 0.5 \\
\hline$l_{4}$ & Equally important & $(0.34,0.44,0.56,0.66)$ & 0.7017 \\
\hline$l_{5}$ & More important & $(0.56,0.66,0.75,0.83)$ & 0.8567 \\
\hline$l_{6}$ & Somewhat more important & $(0.75,0.83,0.89,0.95)$ & 0.9583 \\
\hline$l_{7}$ & Much more important & $(0.89,0.95,0.98,1)$ & 1 \\
\hline$l_{8}$ & Absolutely more important & $(1,1,1,1)$ & \\
\hline
\end{tabular}

In order to be calculated, these labels are required to be numerical. Fuzzy numbers are preferred to represent them, as shown in column 3 , Table 1 . The trapezoid fuzzy number $(\mathrm{TFN}) t=(a, b, c, d)$ is used, and its value $V(t)$ and ambiguity $A(t)$ are real numbers:

$$
\begin{aligned}
& V(t)=\frac{c+b}{2}+\frac{(d-c)-(b-a)}{6} . \\
& A(t)=\frac{c-b}{2}+\frac{(d-c)+(b-a)}{6} .
\end{aligned}
$$

The TFNs would be compared by 


$$
t \mathrm{f} t^{\prime} \Leftrightarrow\left\{\begin{array}{l}
V(t)>V\left(t^{\prime}\right) \\
o r \\
V(t)=V\left(t^{\prime}\right) \text { and } \quad A(t)<A\left(t^{\prime}\right) .
\end{array}\right.
$$

To make decision in linguistic context, two different linguistic Borda counts, broad and narrow, are introduced. The broad means the aggregation of all the preference levels, while the narrow means only counting those greater than the central label $l_{\mathrm{s} / 2}$. Therefore, the broad Borda count of agent $k$ about factor $i$ is defined as

$$
\bar{r}_{k}\left(x_{i}\right)=\sum_{j=1}^{n} r_{i j}^{k} \text {. }
$$

where $r_{i j}^{k}$ is the relative significance of factor $i$ over $j$ given by agent $k$. And the narrow Borda count is defined as

$$
\hat{r}_{k}\left(x_{i}\right)=\sum_{x_{i} \mathrm{f}_{k} x_{j}} r_{i j}^{k}
$$

Consequently, the collective broad Borda count of factor $i$ is defined by

$$
\bar{r}\left(x_{i}\right)=\sum_{k=1}^{m} \bar{r}_{k}\left(x_{i}\right) \text {. }
$$

and the collective narrow Borda count is defined by

$$
\hat{r}\left(x_{i}\right)=\sum_{k=1}^{m} \hat{r}_{k}\left(x_{i}\right) \text {. }
$$

Obviously, the above definitions are based on the assumption that all the agents' opinions are taken equally, and every agent knows this filed pretty well so that he can give his judgment fully confidently. While in realty, because of different social positions, backgrounds, knowledge and experiences, the agents cannot always provide an assuring answer, and they cannot be treated equally. Therefore, here a confidence index $c \in[0,1]$ and an importance index $p \in[0,1]$ are introduced to represent the extent to which the agents' decisions are to be taken into consideration. Accordingly, the Eq. (4) (7) become $(8) \sim(11)$, respectively:

$$
\begin{aligned}
& \bar{r}_{k}\left(x_{i}\right)=\sum_{j=1}^{n} c_{i j}^{k} \cdot r_{i j}^{k} . \\
& \hat{r}_{k}\left(x_{i}\right)=\sum_{x_{i} \mathrm{f}_{k} x_{j}} c_{i j}^{k} \cdot r_{i j}^{k} . \\
& \bar{r}\left(x_{i}\right)=\sum_{k=1}^{m} p_{k} \cdot \bar{r}_{k}\left(x_{i}\right) . \\
& \hat{r}\left(x_{i}\right)=\sum_{k=1}^{m} p_{k} \cdot \hat{r}_{k}\left(x_{i}\right)
\end{aligned}
$$


where $c_{i j}^{k}$ refers to the confidence degree to which the agent $k$ compares $x_{i}$ to $x_{j}$, and clearly, $c_{j i}^{k}=c_{i j}^{k} ; p_{k}$ means the weight of agent $k$ in group decision making.

Similar to the assessment labels, confidence is represented in five degrees listed in Table 2:

Table 2 Grade of confidence

\begin{tabular}{|c|l|l|r|}
\hline Degree & \multicolumn{1}{|c|}{ Meaning } & \multicolumn{1}{c|}{ TFN } & \multicolumn{1}{c|}{$V(t)$} \\
\hline$d_{1}$ & Not confident & $(0,0.1,0.2,0.3)$ & 0.15 \\
\hline$d_{2}$ & Somewhat confident & $(0.2,0.3,0.4,0.5)$ & 0.35 \\
\hline$d_{3}$ & Confident & $(0.4,0.5,0.7,0.8)$ & 0.6 \\
\hline$d_{4}$ & Very confident & $(0.7,0.8,0.9,1)$ & 0.85 \\
\hline$d_{5}$ & Absolutely confident & $(1,1,1,1)$ & 1 \\
\hline
\end{tabular}

Because the linguistic broad Borda count presents better properties than the narrow [18], the former will be adopted in the following.

\section{Deriving Weights Based on Borda Count}

After broad Borda counts are obtained, the weights of criteria can be derived. Detailed steps include:

(1) To form decision matrices

Suppose there are $\mathrm{m}$ agents to assert the weights of $\mathrm{n}$ criteria. Each one $k \in\{1, \mathrm{~K}, m\}$ compares all the pairs of $X\left(X=\left\{x_{i} \mid i \in\{1, \mathrm{~K}, n\}\right\}\right)$ and declares levels of preference by means of a linguistic binary relation $R^{k}: X \times X \rightarrow L$. Therefore $\mathrm{m}$ assertion matrices are formed:

$$
R^{k}=\left(\begin{array}{l}
r_{11}^{k}, \ldots, r_{1 n}^{k} \\
\mathrm{~L} \mathrm{~L} \mathrm{~L} \\
r_{n 1}^{k} \mathrm{~K}, r_{n n}^{k}
\end{array}\right),(k=1, \mathrm{~K} m)
$$

where $r_{i j}^{k}$ takes the form of linguistic label (as shown in Table 1), meaning the level of preference with which agent $k$ prefers $x_{\mathrm{i}}$ over $x_{\mathrm{j}}$. The matrices satisfy the following reciprocity condition:

$$
r_{i j}^{k}=l_{h} \Leftrightarrow r_{j i}^{k}=l_{s-h} .
$$

(2) To get the broad Borda count of each criterion by agent $k$ with Eq. (8);

(3) To calculate the collective broad Borda count of each criterion with Eq. (10), aggregating all the respondents opinions. Then the criteria can be ordered by the value of their Borda counts.

(4) To obtain the criteria weights. Because it is only the preference order that can be obtained by Borda count, more efforts have to be made to get the criteria weights. According to the original definition of Borda count $[11,12,13,14]$, Borda count refers to the number of alternatives that is behind the specific one in a priority ordering. That is, if there are $n$ criteria in a descending order, the Borda count of the 1 st is $(n-1)$, the $2 \mathrm{nd}$ is $(n-2)$, and so on. Therefore, the ideal/maximum Borda count of an alternative should be $(n-1)$. And the ratio of real Borda count to the maximum one can be taken as the assessment of the relative importance of an alternative. Then the non-uniformed $W_{i}$ could be defined as:

$$
W_{i}=\frac{\bar{r}\left(x_{i}\right)}{n-1}
$$


where $\bar{r}\left(x_{i}\right)$ is the broad Borda count obtained in step (3) by Eq. (10). After uniformed, the final weights can be obtained.

\section{Example}

Conservation buffers are a structural mixture of vegetative strips consisting of selected trees, shrubs and grasses placed in landscape. Well established conservation buffers in landscape generally have multiple functions and/or benefits. The well recognized functions and/or benefits include controlling soil erosion, reducing surface runoff and runoff-related pollutants, enhancing wildlife habitat and mitigating stormwater impacts. Various federal, state and local programs provide funding to support the placement of conservation buffers. Conservation buffers should be strategically placed in landscape to maximize those functions and/or benefits and therefore increase the effectiveness of the program funding for conservation buffer placement and maintenance [19].

A survey was conducted, whose aim is to assess how various factors including the expected functions and/or benefits will affect the placement of conservation buffers in landscape. Fifteen experts/agents $(k=15)$ are asked to compare the degree of significance of five factors $(n=5)$ in making decisions regarding the placement of conservation buffers in landscapes by finishing the statements in a questionnaire. In addition to comparing the degree of the importance of the factors between any two selected factors, experts are also asked to indicate the level of confidence in making their ranking in a 1-5 scale as indicated in Table 2.

According to the responded questionnaires, the experts' opinions can be transformed into the form of Eq. (12), taking the 1st expert as an example:

$$
R^{1}=\left(\begin{array}{lllll}
l_{4}, & l_{4}, & l_{2}, & l_{3}, & l_{3} \\
l_{4}, & l_{4}, & l_{1}, & l_{4}, & l_{3} \\
l_{6}, & l_{7}, & l_{4}, & l_{5}, & l_{3} \\
l_{5}, & l_{4}, & l_{3}, & l_{4}, & l_{3} \\
l_{5}, & l_{5}, & l_{5}, & l_{5}, & l_{4}
\end{array}\right)
$$

where $l_{\mathrm{i}}$ is a linguistic label and has the meaning shown in Table 1.

And the confidence of the 1st expert while making this paired comparison is

$$
C^{1}=\left(\begin{array}{lllll}
d_{5}, & d_{4}, & d_{4}, & d_{3}, & d_{3} \\
d_{4}, & d_{5}, & d_{4}, & d_{3}, & d_{3} \\
d_{4}, & d_{4}, & d_{5}, & d_{4}, & d_{3} \\
d_{3}, & d_{3}, & d_{4}, & d_{5}, & d_{3} \\
d_{3}, & d_{3}, & d_{3}, & d_{3}, & d_{5}
\end{array}\right)
$$

Then calculate broad Borda counts of factors by Eq. (8) wherein labels' values listed in Table 1:

$$
\bar{R}^{1}=\left(\begin{array}{l}
d_{5} \cdot l_{4}+d_{4} \cdot l_{4}+d_{4} \cdot l_{2}+d_{3} \cdot l_{3}+d_{3} \cdot l_{3} \\
d_{4} \cdot l_{4}+d_{5} \cdot l_{4}+d_{4} \cdot l_{1}+d_{3} \cdot l_{4}+d_{3} \cdot l_{3} \\
d_{4} \cdot l_{6}+d_{4} \cdot l_{7}+d_{5} \cdot l_{4}+d_{4} \cdot l_{5}+d_{3} \cdot l_{3} \\
d_{3} \cdot l_{5}+d_{3} \cdot l_{4}+d_{4} \cdot l_{3}+d_{5} \cdot l_{4}+d_{3} \cdot l_{3} \\
d_{3} \cdot l_{5}+d_{3} \cdot l_{5}+d_{3} \cdot l_{5}+d_{3} \cdot l_{5}+d_{5} \cdot l_{4}
\end{array}\right)=\left(\begin{array}{c}
1.405 \\
1.439 \\
2.818 \\
1.654 \\
2.184
\end{array}\right)
$$


Deal with other assessment matrices in this way to get $\bar{R}^{k}(k=1, \mathrm{~K} 15)$. Then aggregate the experts' opinions by Eq. (10), assuming the weights of experts $p_{k}(k=1, \mathrm{~K} 15)$ are already given as $(0.03,0.08$, $0.05,0.09,0.07,0.09,0.05,0.03,0.06,0.08,0.05,0.06,0.11,0.07,0.09)$, and the final result is $\bar{R}=(1.650,1.698,2.703,2.308,2.528)$.

Therefore, the order of factors in importance is: $x_{3} \mathrm{f} x_{5} \mathrm{f} x_{4} \mathrm{f} x_{2} \mathrm{f} x_{1}$.

The weights can be obtained by Eq. (14), as tabulated in Table 3. To make a comparison, the weights gained by disregarding experts' confidence (that is, $\mathrm{C}^{\mathrm{k}}$ is a full-one matrix) are also listed there. It can be learnt that the experts' confidence increases the value of each weight (non-uniformed weight of each criterion is increased), but the relative importance between weights has little relationship with experts' confidence (uniformed weights almost keep the same for each criterion).

Table 3 Weights of five criteria in descending order

\begin{tabular}{|l|c|c|c|c|c|c|c|c|c|c|}
\hline & \multicolumn{4}{|c|}{ Considering Confidence } & \multicolumn{4}{c|}{ Not Considering Confidence } \\
\hline Criteria number & 3 & 5 & 4 & 2 & 1 & 3 & 5 & 4 & 2 & 1 \\
\hline Non-uniformed weights & 0.68 & 0.63 & 0.58 & 0.43 & 0.41 & 0.79 & 0.75 & 0.65 & 0.49 & 0.47 \\
\hline Uniformed weights & 0.25 & 0.23 & 0.21 & 0.16 & 0.15 & 0.25 & 0.24 & 0.21 & 0.16 & 0.14 \\
\hline
\end{tabular}

\section{Conclusion}

An improved method to apply fuzzy Borda count in Group Multi-Criteria Decision Making (GMCDM) is presented in this paper, which takes the agents' confidence and importance into account while aggregating their opinions, and then derives the weights from a priority order. The most advantage of this method is that a consistency check, which is necessary in AHP, could be avoided, which saves more times of judgment. Additionally, it can be used in linguistic context and the calculation of Borda count makes the process of decision easier to be realized by programming.

\section{References}

[1] R.R. Yager: An approach to ordinal decision making, International Journal of Approximate Reasoning Vol. 12 (1995), p. 237-261.

[2] F. Herrera, E. Herrera-Viedma, J.L. Verdegay: A rational consensus model in group decision making using linguistic assessments, Fuzzy Sets and Systems Vol. 88 (1997), p. 31-49.

[3] V. Torra: The weighted OWA operator, International Journal of Intelligent Systems Vol. 12 (1997), p. 153-166.

[4] M. Zarghami, F. Szidarovszky: Stochastic-fuzzy multi criteria decision making for robust water resources management, Stochastic Environmental Research and Risk Assessment Vol. 23 (2009), p. 329-339.

[5] J. McPhee, W.W.G. Yeh: Multiobjective optimization for sustainable groundwater management in semiarid regions, Journal of Water Resources Planning and Management Vol. 130 (2004), p. 490-497.

[6] D. Stroppiana, M. Boschetti, P.A. Brivio, P. Carrara, G. Bordogna: A fuzzy anomaly indicator for environmental monitoring at continental scale, Ecological Indicators Vol. 9 (2009), p. 92-106.

[7] Z. Xu: An overview of methods for determining OWA weights, International Journal of Intelligent Systems Vol. 20 (2005), p. 843-865.

[8] T.L. Saaty: The analytic hierarchy process (McGraw-Hill, New York 1980).

[9] T.L. Saaty: A scaling method for priorities in hierarchical structures, Journal of Mathematical Psychology Vol. 15 (1977), p. 234-281. 
[10] M. Dummett: The Borda count and agenda manipulation, Social Choice and Welfare Vol. 15 (1998), p. 289- 296.

[11] D. Black: The Theory of Committees and Elections (Kluwer Academic Publishers, Boston 1958).

[12] D. Black: Partial justification of the Borda count, Public Choice Vol. 28 (1976), p. 1- 16.

[13] D.C. Mueller: Public Choice (Cambridge University Press, London 1979).

[14] J.C. de Borda: Mémoire sur les élections au scrutin (Historie de l'Academie Royale des Sciences, Paris 1784).

[15] P. Gärdenfors: Positionalist voting functions, Theory and Decision Vol. 4 (1973), p. 1- 24.

[16] Hannu Nurmi: Resolving Group Choice Paradoxes Using Probabilistic and Fuzzy Concepts, Group Decision and Negotiation Vol. 10 (2001), p. 177-199.

[17] J.L. García-Lapresta, M. Martínez-Panero: Borda Count Versus Approval Voting - A Fuzzy Approach, Public Choice Vol. 112 (2002), p.167-184.

[18] J.L. García-Lapresta, M. Martínez-Panero, L.C. Meneses: Defining the Borda count in a linguistic decision making context, Information Sciences Vol. 179 (2009), p. 2309- 2316.

[19] Z. Qiu, M.G. Dosskey: Multiple function benefit - Cost comparison of conservation buffer placement strategies, Landscape and Urban Planning Vol. 107 (2012), p. 89- 99. 